\title{
EXACT BOUNDS FOR THE STOCHASTIC UPWARD MATCHING PROBLEM
}

\author{
WANSOO T. RHEE AND MICHEL TALAGRAND
}

\begin{abstract}
We draw at random independently and according to the uniform distribution two sets of $n$ points of the unit square. We consider a maximum matching of points of the first set with points of the second set with the restriction that a point can be matched only with a point located at its upper right. Then with probability close to one, the number of unmatched points is of order $n^{1 / 2}(\log n)^{3 / 4}$.
\end{abstract}

1. Introduction. Consider $2 n$ points $X_{1}, \ldots, X_{n}, Y_{1}, \ldots, Y_{n}$ of the unit square. An upward matching is a one-to-one map $\phi$ from a subset $I$ of $\{1, \ldots, n\}$ to $\{1, \ldots, n\}$ such that for $i$ in $I$, each coordinate of $Y_{\phi(i)}$ is greater than the corresponding coordinate of $X_{i}$. The matching is called maximum if the cardinality of $I$ is maximum. A point $X_{i}$ is called unmatched if $i \notin I$. We are interested here in the stochastic version of the problem, where $X_{1}, \ldots, X_{n}, Y_{1}, \ldots, Y_{n}$ are independent and uniformly distributed over the unit square, and we want to estimate the number of unmatched points in a maximum upward matching (that we will call for short "the number of unmatched points"). Interesting connections have been recently discovered between this question and the probabilistic analysis of some bin-packing algorithms $[\mathbf{6}, \mathbf{8}]$. The bound we give here also provide improved bounds on the expected performance of these algorithms.

A subset $C$ of the unit square is called a lower class if it is closed and if it contains $(x, y)$ whenever it contains some point $\left(x^{\prime}, y^{\prime}\right)$ with $x^{\prime} \geq x, y^{\prime} \geq y$. We denote by $\mathcal{L}$ the collection of all lower classes. A consequence of the Marriage Lemma is that the number $M_{n}(X, Y)$ of unmatched points is equal to

$$
\operatorname{Sup}_{C \in \mathcal{L}}\left(\operatorname{Card}\left\{i \leq n ; Y_{i} \in C\right\}-\operatorname{Card}\left\{i \leq n ; X_{i} \in C\right\}\right)
$$

Let us now explain another point of view. For a point $x$, we denote by $\delta_{x}$ the point mass at $x$. So, for any set $C, \delta_{x}(C)=1$ if $C$ contains $x, \delta_{x}(C)=0$ otherwise. Denote by $\lambda$ the uniform probability on the unit square. For a $n$-tuple

Received by the editors April 1, 1986. $60 \mathrm{G} 17$.

1980 Mathematics Subject Classification (1985 Revision). Primary 60D05; Secondary 60B10,

Key words and phrases. Empirical processes, empirical discrepancy, majorizing measures, lower classes, bin packing. 
$X=\left(X_{1}, \ldots, X_{n}\right)$ of the unit square, let

$$
\begin{aligned}
D_{n}(X) & =\operatorname{Sup}_{C \in \mathcal{L}}\left(\left|\frac{1}{n} \sum_{i \leq n} \delta_{X_{i}}(C)-\lambda(C)\right|\right) \\
& =\operatorname{Sup}_{C \in \mathcal{L}}\left(\left|\frac{1}{n} \operatorname{card}\left\{i ; i \leq n, X_{i} \in C\right\}-\lambda(C)\right|\right) .
\end{aligned}
$$

So $D_{n}(X)$ measures how well the empirical measure $(1 / n) \sum_{i \leq n} \delta_{X_{i}}$ approximates $\lambda$ uniformly over the class $\mathcal{L}$. The quantity $D_{n}(X)$ is usually called the empirical discrepancy relative to $\mathcal{L}$. The estimation of the empirical discrepancy relative to a given class of sets is one of the objects of the theory of empirical processes. The relation with our problem is that $M_{n}(X, Y) \leq n\left(D_{n}(X)+D_{n}(Y)\right)$. (It is easy to see that actually the problems of estimating the size of $M_{n}$ and of $D_{n}$ are essentially equivalent.) The empirical discrepancy $D_{n}(X)$ has been studied by R. M. Dudley $[\mathbf{3}, \mathbf{4}]$. He proved that for each $\delta>0$, there is a constant $K_{\delta}$ such that

$$
\lim _{n \rightarrow \infty} P\left(D_{n}(X) \geq K_{\delta} n^{-1 / 2}(\log n)^{1 / 2}(\log \log n)^{-\delta}\right)=1 .
$$

In an independent effort, R. Karp, M. Luby and A. Marchetti-Spaccamela [6] proved that for some constants $K_{1}, K_{2}$, we have

$$
\lim _{n \rightarrow \infty} P\left(K_{1}(n \log n)^{1 / 2} \leq M_{n}(X, Y) \leq K_{2} n^{1 / 2} \log n\right)=1 .
$$

This lower bound was improved by P. Shor $[\mathbf{8}]$, who proved that

$$
\lim _{n \rightarrow \infty} P\left(M_{n}(X, Y) \geq K_{1} n^{1 / 2}(\log n)^{3 / 4}\right)=1 .
$$

Our result is that this lower bound is of the right order.

THEOREM A. For some constants $K_{1}, K_{2}$,

$$
\begin{aligned}
& \lim _{n \rightarrow \infty} P\left(K_{1} n^{1 / 2}(\log n)^{3 / 4} \leq M_{n}(X, Y) \leq K_{2} n^{1 / 2}(\log n)^{3 / 4}\right)=1 . \\
& \lim _{n \rightarrow \infty} P\left(K_{1} n^{-1 / 2}(\log n)^{3 / 4} \leq D_{n}(X) \leq K_{2} n^{-1 / 2}(\log n)^{3 / 4}\right)=1 .
\end{aligned}
$$

Our discussion, and the result of $\mathrm{P}$. Shor, show that it is enough to prove that

$$
\lim _{n \rightarrow \infty} P\left(D_{n}(X) \leq K_{2} n^{-1 / 2}(\log n)^{3 / 4}\right)=1 .
$$

Our proof will make use of some very fine points of the theory of stochastic processes. We believe that this is the nature of the problem and cannot be avoided. The following problem of interest remains open, and actually seems far beyond the range of the methods of the present work.

Problem. Does there exist a constant $C$ such that

$$
\lim _{n \rightarrow \infty} D_{n}(X) n^{1 / 2}(\log n)^{-3 / 4}=C \quad \text { a.s.? }
$$

After this work had been completed, the authors learned that their main result has been obtained independently and somewhat earlier by F. T. Leighton and P. Shor [7] using completely different methods. 
2. Preparation. We will denote by $K_{0}, K_{1}, K_{2}, \ldots$ universal constants. When there is no point to distinguish between the various constants, we just denote by $K$ a universal constant, not necessarily the same at each line. We make no attempt to produce sharp constants; our methods do not lend themselves to this; so we always use simple estimates (however crude) whenever possible.

We fix $n$, and we let $q$ be the largest integer such that $2^{-q} \geq n^{-1 / 2}(\log n)^{3 / 4}$, so $2^{-q} \leq 2 n^{-1 / 2}(\log n)^{3 / 4}$. We can suppose $n$ large enough that $q \geq 1$. We have $K_{1} \log n \leq q \leq K_{2} \log n$. For $p \leq q$, we consider

$$
A_{p}=\left\{k 2^{-p} ; 0<k<2^{p}\right\}
$$

so $\operatorname{card} A_{p}=2^{p}-1$.

Denote by $\mathcal{F}_{q}$ the set of nonincreasing functions $u$ from $[0,1]$ to $[0,1]$ that are constants on each interval $\left.](k-1) 2^{-q}, k 2^{-q}\right]$ for $1 \leq k<2^{-q}$ and equal to zero on ] $\left.1-2^{-q}, 1\right]$. For $u$ in $\mathcal{F}_{q}$, the lower graph

$$
C(u)=\{(x, y) ; 0 \leq x \leq 1,0 \leq y \leq u(x)\}
$$

is a lower class. We denote by $\mathcal{L}_{q}$ the collection of these lower graphs. We denote by $\mathcal{L}_{q}^{\prime}$ the subcollection of the lower graphs of those functions $u$ in $\mathcal{F}_{q}$ that take only values of the type $k 2^{-p}, 0 \leq k \leq 2^{p}$. We fix a subset $T$ of $\mathcal{L}_{q}$ with the following properties:

Whenever $C_{1}, C_{2}$ belong to $T, C_{1} \neq C_{2}$, we have

$$
\lambda\left(C_{1} \triangle C_{2}\right)>8\left(2^{-q}\right) \text {. }
$$

Whenever $C$ belongs to $\mathcal{L}$, there is $C^{\prime}$ in $T$ such that

$$
\lambda\left(C \triangle C^{\prime}\right) \leq 9\left(2^{-q}\right) .
$$

(We note that a set of largest possible cardinality that satisfies (4) also satisfies (5). Indeed one sees that $\lambda\left(C \triangle C_{1}\right) \leq 2^{-q}$ for some $C_{1}$ in $\mathcal{L}_{q}$, and that $\lambda\left(C_{1} \Delta C^{\prime}\right) \leq$ $8\left(2^{-q}\right)$ for some $C^{\prime}$ in $T$ by the maximality of $T$.) We also note the following, that is a consequence of well known estimates on the tail of the binomial distribution. For any set $C$ of the unit square, we have

$$
P\left(\frac{1}{n} \sum_{i \leq n} \delta_{X_{i}}(C) \geq 2 \lambda(C)\right) \leq \exp \left(\frac{-n \lambda(C)}{3}\right) .
$$

LEMMA 1. There exist $\alpha>0$ and $K>0$ such that for $n$ large enough, with probability $\geq 1-\exp \left(-\alpha n^{1 / 2}(\log n)^{3 / 4}\right)$, the following occur.

(a) Whenever $C_{1}, C_{2} \in \mathcal{L}$, we have

$$
\frac{1}{n} \sum_{i \leq n} \delta_{X_{i}}\left(C_{1} \triangle C_{2}\right) \leq 8\left(2^{-q}\right)+2 \lambda\left(C_{1} \triangle C_{2}\right) .
$$

In particular, if $\lambda\left(C_{1} \Delta C_{2}\right) \geq 8\left(2^{-q}\right)$, we have

$$
\frac{1}{n} \sum_{i \leq n} \delta_{X_{i}}\left(C_{1} \triangle C_{2}\right) \leq 3 \lambda\left(C_{1} \Delta C_{2}\right) .
$$

$$
D_{n}(X) \leq K n^{-1 / 2}(\log n)^{3 / 4}+\operatorname{Sup}_{C \in T}\left|\frac{1}{n} \sum_{i \leq n} \delta_{X_{i}}(C)-\lambda(C)\right| .
$$


Proof. For $G_{1}$ and $G_{2}$ in $\mathcal{L}_{q}^{\prime}$, with $G_{1} \subset G_{2}, \lambda\left(G_{2} \backslash G_{1}\right) \geq 2^{-q}$, it follows from (6) that the event

$$
\frac{1}{n} \sum_{i \leq n} \delta_{X_{i}}\left(G_{2} \backslash G_{1}\right) \leq 2 \lambda\left(G_{2} \backslash G_{1}\right)
$$

has a probability at least $1-\exp \left(-n 2^{-q} / 3\right)$. Consider now the event that (7) occurs for all possible choices of $G_{1}, G_{2}$ as above. This has a probability $\geq 1-$ $\left(\operatorname{card} \mathcal{L}_{q}^{\prime}\right)^{2} \exp \left(-n 2^{-q} / 3\right)$. A trivial estimate gives card $\mathcal{L}_{q}^{\prime} \leq\left(2^{q}+1\right)^{2^{q}} \leq \exp \left(2 q 2^{q}\right)$, so

$$
\left(\operatorname{card} \mathcal{L}_{q}^{\prime}\right)^{2} \exp \left(-n 2^{-q} / 3\right) \leq \exp \left(-2^{q}\left(n 2^{-2 q} / 3-4 q\right)\right) .
$$

Since $2^{-q} \geq n^{-1 / 2}(\log n)^{3 / 4}$, we have $n 2^{-2 q} \geq(\log n)^{3 / 2}$. On the other hand, $q \leq K_{2} \log n$; it follows that for some $\alpha>0$, and for $n$ large enough, we have $2^{q}\left(n 2^{-2 q} / 3-4 q\right) \geq \alpha n^{1 / 2}(\log n)^{3 / 4}$. We now prove that (a) and (b) hold whenever (7) holds for all possible choices of $G_{1}$ and $G_{2}$ as above.

We first observe that for each $C$ in $\mathcal{L}$, there exist $C^{\prime}$ and $C^{\prime \prime}$ in $\mathcal{L}_{q}$ such that $C^{\prime} \subset C \subset C^{\prime \prime}$ and that $\lambda\left(C^{\prime \prime} \backslash C^{\prime}\right) \leq 2^{-q}$. So, given $C_{1}$ and $C_{2}$ in $\mathcal{L}$, we can find $C_{1}^{\prime}, C_{1}^{\prime \prime}, C_{2}^{\prime}, C_{2}^{\prime \prime}$ in $\mathcal{L}_{q}^{\prime}$ such that $C_{1} \triangle C_{2} \subset\left(C_{2}^{\prime \prime} \backslash C_{1}^{\prime}\right) \cup\left(C_{1}^{\prime \prime} \backslash C_{2}^{\prime}\right)$ and

$$
\lambda\left(C_{2}^{\prime \prime} \backslash C_{1}^{\prime}\right) \leq 2^{-q+1}+\lambda\left(C_{2} \backslash C_{1}\right) ; \quad \lambda\left(C_{1}^{\prime \prime} \backslash C_{2}^{\prime}\right) \leq 2^{-q+1}+\lambda\left(C_{1} \backslash C_{2}\right) .
$$

It is easy to see that one can if necessary decrease $C_{1}^{\prime}$ and $C_{2}^{\prime}$ and increase $C_{2}^{\prime \prime}$ and $C_{1}^{\prime \prime}$ to achieve

$$
2^{-q} \leq \lambda\left(C_{2}^{\prime \prime} \backslash C_{1}^{\prime}\right) \leq 2^{-q+1}+\lambda\left(C_{2} \backslash C_{1}\right)
$$

and

$$
2^{-q} \leq \lambda\left(C_{1}^{\prime \prime} \backslash C_{2}^{\prime}\right) \leq 2^{-q+1}+\lambda\left(C_{1} \backslash C_{2}\right)
$$

so

$$
\begin{aligned}
\lambda\left(C_{2}^{\prime \prime} \backslash C_{1}^{\prime}\right)+\lambda\left(C_{1}^{\prime \prime} \backslash C_{2}^{\prime}\right) & \leq 4\left(2^{-q}\right)+\lambda\left(C_{2} \backslash C_{1}\right)+\lambda\left(C_{1} \backslash C_{2}\right) \\
& \leq 4\left(2^{-q}\right)+\lambda\left(C_{1} \triangle C_{2}\right) .
\end{aligned}
$$

It follows that

$$
\begin{aligned}
\frac{1}{n} \sum_{i \leq n} \delta_{X_{i}}\left(C_{1} \Delta C_{2}\right) & \leq \frac{1}{n} \sum_{i \leq n} \delta_{X_{i}}\left(C_{2}^{\prime \prime} \backslash C_{1}^{\prime}\right)+\frac{1}{n} \sum_{i \leq n} \delta_{X_{i}}\left(C_{1}^{\prime \prime} \backslash C_{2}^{\prime}\right) \\
& \leq 2\left(\lambda\left(C_{2}^{\prime \prime} \backslash C_{1}^{\prime}\right)+\lambda\left(C_{1}^{\prime \prime} \backslash C_{2}^{\prime}\right)\right) \leq 8\left(2^{-q}\right)+2 \lambda\left(C_{1} \Delta C_{2}\right) .
\end{aligned}
$$

This proves (a).

To prove (b), we note that given a lower set $C$, there is $C_{1}$ in $T$ such that $\lambda\left(C \triangle C_{1}\right) \leq 9\left(2^{-q}\right)$. By (a), we have

$$
\begin{aligned}
\left|\frac{1}{n} \sum_{i \leq n} \delta_{X_{i}}(C)-\lambda(C)\right| \leq & \left|\frac{1}{n} \sum_{i \leq n} \delta_{X_{i}}\left(C_{1}\right)-\lambda\left(C_{1}\right)\right| \\
& +\frac{1}{n} \sum_{i \leq n} \delta_{X_{i}}\left(C \triangle C_{1}\right)+\lambda\left(C \triangle C_{1}\right) \\
\leq & \left|\frac{1}{n} \sum_{i \leq n} \delta_{X_{i}}\left(C_{1}\right)-\lambda\left(C_{1}\right)\right|+35\left(2^{-q}\right) .
\end{aligned}
$$


So,

$$
D_{n}(X) \leq \operatorname{Sup}_{C \in T}\left|\frac{1}{n} \sum_{i \leq n} \delta_{X_{i}}(C)-\lambda(C)\right|+43\left(2^{-q}\right),
$$

and $35\left(2^{-q}\right) \leq 70 n^{-1 / 2}(\log n)^{3 / 4}$. This completes the proof.

We are now faced with the problem of estimating

$$
\operatorname{Sup}_{C \in T}\left|\frac{1}{n} \sum_{i \leq n} \delta_{X_{i}}(C)-\lambda(C)\right|,
$$

that is the empirical discrepancy relative to the class $T$. At this point, we must discuss the randomization technique that has been at the core of the recent progress on empirical processes. For clarity, let us denote by $(\Omega, \Sigma, P)$ the basic probability space. Consider another probability space $\left(\Omega^{\prime}, \Sigma^{\prime}, Q\right)$ on which is defined an independent sequence $\left(\varepsilon_{i}\right)_{i \leq n}$ of random variables (r.v.) with $Q\left(\varepsilon_{i}=1\right)=$ $Q\left(\varepsilon_{i}=-1\right)=0$. Let us denote by $\operatorname{Pr}$ the product probability on $\Omega \times \Omega^{\prime}$. We can now consider the r.v. on $\Omega \times \Omega^{\prime}$ defined by

$$
\operatorname{Sup}_{C \in T}\left|\frac{1}{n} \sum_{i \leq n} \varepsilon_{i}(\omega) \delta_{X_{i}}(C)\right| .
$$

A very useful fact is that the behavior of this random variable is closely connected to that of the empirical discrepancy. We will need in particular the following (easy) fact.

LEMma $2[5,2.7, \mathrm{~b}]$. For $t>n^{-1 / 2}$, we have

$$
\begin{aligned}
& P\left(\operatorname{Sup}_{C \in T}\left|\frac{1}{n} \sum_{i \leq n} \delta_{X_{i}}(C)-\lambda(C)\right|>t\right) \\
& \quad \leq \operatorname{Pr}\left(\operatorname{Sup}_{C \in T}\left|\frac{1}{n} \sum_{i \leq n} \varepsilon_{i}(\omega) \delta_{X_{i}}(C)\right|>\frac{1}{2}\left(t-n^{-1 / 2}\right)\right) .
\end{aligned}
$$

We now study the quantity (8). Assume that $X_{1}, \ldots, X_{n}$ are given and satisfy (a) and (b) of Lemma 1. For $C$ in $T$, consider the random variable $Y_{C}$ on $\Omega^{\prime}$ given by

$$
Y_{C}=\frac{1}{\sqrt{n}} \sum_{i \leq n} \varepsilon_{i}(\omega) \delta_{X_{i}}(C)
$$

We have to estimate $\operatorname{Sup}_{C \in T}\left|Y_{C}(\omega)\right|$. To explore the properties of the variables $Y_{C}$, we recall the well-known inequality:

$$
\text { For } t>0, \quad Q\left(\left|\sum_{i \leq k} \varepsilon_{i}(\omega)\right| \geq t\right) \leq 2 \exp \left(\frac{-t^{2}}{2 k}\right) .
$$

This can be considered as an inequality on the tails of the binomial distribution. For a proof, see e.g. [5, p. 942]. 
For $C_{1}, C_{2}$ in $T$, we have

$$
Y_{C_{1}}-Y_{C_{2}} \stackrel{D}{=} \frac{1}{\sqrt{n}} \sum_{i \in J} \varepsilon_{i}(\omega)
$$

where $J=\left\{i \leq n ; X_{i} \in C_{1} \Delta C_{2}\right\}$. So, by (a) of Lemma 1 , we have card $J \leq$ $3 n \lambda\left(C_{1} \triangle C_{2}\right)$. It follows from (9) that

$$
Q\left(\left|Y_{C_{1}}-Y_{C_{2}}\right| \geq t\right) \leq 2 \exp \left(-t^{2} / 6 \lambda\left(C_{1} \triangle C_{2}\right)\right) .
$$

Consider now the distance $d$ on $T$ given by $d\left(C_{1}, C_{2}\right)=\left(\lambda\left(C_{1} \triangle C_{2}\right)\right)^{1 / 2}$. We can rewrite (10) as

$$
Q\left(\left|Y_{C_{1}}-Y_{C_{2}}\right| / d\left(C_{1}, C_{2}\right) \geq t\right) \leq 2 \exp \left(-t^{2} / 6\right) .
$$

So, the tails of the r.v. $\left(Y_{C_{1}}-Y_{C_{2}}\right) / d\left(C_{1}, C_{2}\right)$ are similar to the tails of the Gaussian distribution. We can now view the situation as follows: We have a (finite) metric space $(T, d)$, and r.v. $\left(Y_{C}\right)_{C \in T}$ that satisfy (11). How do we estimate the r.v. $\operatorname{Sup}_{C \in T}\left|Y_{C}\right|$ ? This problem is closely related to the theory of Gaussian processes, and thus has received much study. The best known result in that direction is due to R. M. Dudley [1] (in the Gaussian case). For $i \geq 1$, denote by $N(i)$ the smallest number of $d$ balls of radius $2^{-i}$ needed to cover $T$. Then in our case Dudley's result yields (fixing $C_{0}$ in $T$ )

$$
E \operatorname{Sup}_{C \in T}\left|Y_{C}-Y_{C_{0}}\right| \leq K \sum_{i=1}^{\infty} 2^{-i}(\log N(i))^{1 / 2},
$$

for some universal constant $K$. Simple estimates show that this sum is of order $q$, i.e. of order $\log n$. Use of this method then gives a bound on $M_{n}(X, Y)$ of order $n^{-1 / 2} \log n$, that is the bound found by R. Karp, M. Luby, and A. MarchettiSpaccamela [6]. This is not surprising, since analysis of their proof reveals that it is based on a principle very similar to the ideas behind Dudley's theorem. To get a better bound, we have to use more precise tools to estimate $\operatorname{Sup}_{T}\left|Y_{C}\right|$. The right tools are due, in their final form, to X. Fernique (after essential contributions by C. Preston and others) and are called majorizing measures [4]. Until recently, these objects have been generally considered, even by the specialists, as mere curiosities. They have however recently turned out to be of importance since they are the right tool to understand general Gaussian processes. (The insight gained in [9] was essential to the completion of the present work.) As far as we know, the present work is the first time that majorizing measures are applied to a "concrete" problem. Here is the version of X. Fernique's result that we need. For convenience of notations, we set throughout the paper $h(t)=(\log 1 / t)^{1 / 2}$ for $0<t \leq 1$. We note that $h$ is decreasing.

LEMMA 3. Consider a finite metric space $(T, d)$. For $u$ in $T$, consider a r.v. $Y_{u}$. Let $\beta>0$. Assume that for $u$ and $v$ in $T, u \neq v$, we have

$$
\text { for } t>0, \quad P\left(\left|Y_{u}-Y_{v}\right| / d(u, v) \geq t\right) \leq 2 \exp \left(-\beta t^{2}\right) \text {. }
$$

For $x$ in $T$, denote by $B(x, \varepsilon)$ the ball $\{y \in T, d(x, y) \leq \varepsilon\}$. Let $l$ be large enough that $B\left(x, 2^{-l}\right)=\{x\}$ for each $x$ in $T$. Assume that $d(x, y) \leq 1$ whenever $x, y \in T$. 
Let $m$ be a probability measure on $(T, d)$. Let $M$ such that

$$
M \geq \operatorname{Sup}_{x \in T} \sum_{i=0}^{l} 2^{-i} h\left(m\left(B\left(x, 2^{-i}\right)\right)\right) .
$$

Then, for some universal constant $K$, we have

$$
P\left(\operatorname{Sup}_{u, v \in T}\left|Y_{u}-Y_{v}\right|>K M \beta^{-1 / 2}\right) \leq 2 \exp \left(-M^{2}\right) .
$$

The modifications of Fernique's argument needed to get the present statement are well known by the specialists, but there seems to be no easy reference available, so we will provide a sketch of proof for the convenience of the reader. First, by changing $Y_{u}$ in $\sqrt{2 / \beta} Y_{u}$, we can assume $\beta=2$. The equality

$$
E\left(\exp \left(Z^{2}\right)\right)=\int_{0}^{\infty} 2 t \exp \left(t^{2}\right) P(|Z| \geq t) d t
$$

together with (12) gives

$$
E\left(\exp \left(\left(Y_{u}-Y_{v}\right) / d(u, v)\right)^{2}\right) \leq 2,
$$

whenever $u$ and $v$ are in $T, u \neq v$. Consider the r.v.

$$
I(\omega)=\iint_{T \times T} \exp \left(\frac{Y_{u}(\omega)-Y_{v}(\omega)}{d(u, v)}\right)^{2} d m(u) d m(v)
$$

where we set $\left(Y_{u}(\omega)-Y_{v}(\omega)\right) / d(u, v)=0$ for $u=v$. By $(14)$, we see that $E(I(\omega)) \leq$ 2. The event $I(\omega) \geq \exp \left(M^{2}\right)$ has the probability $\leq 2 \exp \left(-M^{2}\right)$. We show now that when $I(\omega) \leq \exp \left(M^{2}\right)$, we have $\left|Y_{u}(\omega)-Y_{v}(\omega)\right| \leq K M$ for all $u$ and $v$ in $T$. We fix $x$ in $T$. We set, for $1 \leq i \leq l$,

$$
B_{i}=B\left(x, 2^{-i}\right), \quad a_{i}=m\left(B_{i}\right)^{-1} \int_{B_{i}} Y_{u}(\omega) d m(u) .
$$

We have $a_{l}=Y_{x}(\omega)$ and $a_{1}=\int_{T} Y_{u}(\omega) d m(u)$ does not depend on $x$. It is hence enough to show that $\left|a_{1}-a_{l}\right| \leq K M$. Let

$$
f_{i}=m\left(B_{i}\right) m\left(B_{i+1}\right) \exp \left(\left(a_{i+1}-a_{i}\right) / 2^{-i+1}\right)^{2} .
$$

Since the function $t \rightarrow \exp \left(t^{2}\right)$ is convex, we get by Jensen's inequality that

$$
f_{i} \leq \iint_{B_{i+1} \times B_{i}} \exp \left(\frac{Y_{u}(\omega)-Y_{v}(\omega)}{2^{-i+1}}\right)^{2} d m(u) d m(v) .
$$

We note that $d(u, v) \leq 2^{-i+1}$ for $u$ in $B_{i+1}, v$ in $B_{i}$, so $f_{i} \leq I(\omega)$. Letting $b_{i}=m\left(B_{i}\right) m\left(B_{i+1}\right) I(\omega)^{-1}$, we have shown that

$$
\sum_{i=1}^{l-1} 2^{-i+1} b_{i} \exp \left(\frac{a_{i+1}-a_{i}}{2^{-i+1}}\right)^{2} \leq 2 .
$$

We note now that for $x \geq 1, y \geq 0$, we have

$$
x y \leq 2 x(\log x)^{1 / 2}+\exp \left(y^{2}\right) .
$$


Indeed, for $y \leq 2(\log x)^{1 / 2}$, we have $x y \leq 2 x(\log x)^{1 / 2}$ while if $x \leq \exp \left(y^{2} / 4\right)$, we have $x y \leq y \exp \left(y^{2} / 4\right) \leq \exp \left(y^{2}\right)$. Using this for $x_{i}=b_{i}^{-1}, y_{i}=\left|\left(a_{i+1}-a_{i}\right) / 2^{-i+1}\right|$, we get

$$
\begin{aligned}
\left|a_{l}-a_{1}\right| & \leq \sum_{i=1}^{l-1}\left|a_{i+1}-a_{i}\right|=\sum_{i=1}^{l-1} 2^{-i+1} b_{i} x_{i} y_{i} \\
& \leq \sum_{i=1}^{l-1} 2^{-i+1} h\left(b_{i}\right)+2 .
\end{aligned}
$$

The inequality $\left|a_{l}-a_{1}\right| \leq K M$ then follows from (13) and two lines of computation, using the inequality $h(a b) \leq h(a)+h(b)$ and the fact that $(\log I(\omega))^{1 / 2} \leq M$. This completes the proof.

To apply Lemma 3, we must construct an appropriate measure $m$ on $T$ such that the quantity $(13)$ is of order $(\log n)^{3 / 4}$. Estimation of the quantity (13) is unfortunately not an easy task. For a function $u$ in $\mathcal{F}_{q}, p \leq q$ and $\theta \geq 0$, we set

$$
B_{p, q}(u, \theta)=\left\{v \in \mathcal{F}_{q} ; \sum_{a \in A_{p}}|v(a)-u(a)| \leq \theta\right\} .
$$

The main part of our proof is to establish the following statement.

PROPOSITION 4. There exists a probability measure $m_{q}$ on $\mathcal{F}_{q}$ such that for each $u$ in $\mathcal{F}_{q}$, we have

$$
\sum_{i=1}^{q} 2^{-i / 2} h\left(m_{q}\left(B_{i, q}(u, 1)\right)\right) \leq K q^{3 / 4}
$$

where $K$ is a universal constant.

This result will be proved in $\S 3$. We show now how to prove the following that makes more precise our contribution to Theorem A.

THEOREM B. For some universal constants $\alpha, K>0$, we have, for $n$ large enough,

$$
P\left(D_{n}(X) \geq K n^{-1 / 2}(\log n)^{3 / 4}\right) \leq \exp \left(-\alpha(\log n)^{3 / 2}\right) .
$$

We recall that to each $u$ in $\mathcal{F}_{q}$, we associate its lower graph $C(u) \in \mathcal{L}_{q}$. We establish an easy fact.

LEMMA 5. For $v \in B_{p, q}(u, 1)$, we have

$$
\lambda(C(u) \triangle C(v)) \leq 3\left(2^{-p}\right) .
$$

ProOF. We note that $\lambda(C(u) \triangle C(v))=\int_{0}^{1}|u(x)-v(x)| d x$. For $0 \leq k<2^{-p}$, and $x$ in the interval $\left.] k 2^{-p},(k+1) 2^{-p}\right]$, we have

$$
\begin{aligned}
u(x)-v(x) & \leq u\left(k 2^{-p}\right)-v\left((k+1) 2^{-p}\right) \\
& \leq u\left(k 2^{-p}\right)-u\left((k+1) 2^{-p}\right)+\left|u\left((k+1) 2^{-p}\right)-v\left((k+1) 2^{-p}\right)\right|
\end{aligned}
$$

and, similarly,

$$
v(x)-u(x) \leq v\left(k 2^{-p}\right)-v\left((k+1) 2^{-p}\right)+\left|u\left((k+1) 2^{-p}\right)-v\left((k+1) 2^{-p}\right)\right| .
$$


So,

$$
\begin{aligned}
|u(x)-v(x)| \leq & u\left(k 2^{-p}\right)-u\left((k+1) 2^{-p}\right)+v\left(k 2^{-p}\right)-v\left((k+1) 2^{-p}\right) \\
& +\left|u\left((k+1) 2^{-p}\right)-v\left((k+1) 2^{-p}\right)\right| .
\end{aligned}
$$

Since $\sum_{k=1}^{2^{p}}\left|u\left(k 2^{-p}\right)-v\left(k 2^{-p}\right)\right| \leq 1$, the bound $\int_{0}^{1}|u(x)-v(x)| d x \leq 3\left(2^{-p}\right)$ follows by integration over $\left.] k 2^{-p}-(k+1) 2^{-p}\right]$ and summation over $k$.

Since to each $u$ in $\mathcal{F}_{q}$ we associate in a one-to-one way $C(u)$ in $\mathcal{L}_{q}$, we can view the measure $m_{q}$ as being on $\mathcal{L}_{q}$. For $C$ in $\mathcal{L}_{q}, \varepsilon>0$, let

$$
B(C, \varepsilon)=\left\{C^{\prime} \in \mathcal{L}_{q} ; \lambda\left(C \triangle C^{\prime}\right)^{1 / 2} \leq \varepsilon\right\} .
$$

From (17) and Lemma 5, we have, for each $C$ in $\mathcal{L}_{q}$, that

$$
\sum_{i=1}^{q} 2^{-i / 2} h\left(m_{q}\left(B\left(C, 2\left(2^{-i / 2}\right)\right)\right)\right) \leq K q^{3 / 4} .
$$

We are facing a last minor inconvenience. The measure $m_{q}$ is supported by $\mathcal{L}_{q}$, while we are looking for a measure on $T$. This is taken care of in the obvious way, by pushing $m_{q}$ back to $T$. There is a Borel map $\phi$ from $\mathcal{L}_{q}$ to $T$ such that $d(C, \phi(C)) \leq$ $d\left(C, C^{\prime}\right)$ whenever $C^{\prime} \in T$, where, as usual, $d\left(C_{1}, C_{2}\right)=\lambda\left(C_{1} \triangle C_{2}\right)^{1 / 2}$. For any $C$ in $\mathcal{L}_{q}$, there is $C^{\prime}$ in $T$ with $\lambda\left(C \triangle C^{\prime}\right) \leq 9\left(2^{-q}\right)$, so we have $d(C, \phi(C)) \leq 3\left(2^{-q / 2}\right)$. Denote by $m$ the image measure of $m_{q}$ by $\phi$, i.e. $m(U)=m_{q}\left(\phi^{-1}(U)\right)$ for $U \subset T$. For each $C$ in $T$, we have $\phi(B(C, \varepsilon)) \subset B\left(C, \varepsilon+3\left(2^{-q / 2}\right)\right)$. So we have for $i \geq q$, $\phi\left(B\left(C, 2\left(2^{-i / 2}\right)\right)\right) \subset B\left(C, 5\left(2^{-i / 2}\right)\right)$. Since $2^{5 / 2} \geq 5$, we have

$$
m\left(B\left(C, 2^{(-i+5) / 2}\right)\right) \geq m_{q}\left(B\left(C, 2\left(2^{-i / 2}\right)\right)\right) .
$$

It then follows from (18) that for $C$ in $T$,

$$
\sum_{i=1}^{q} 2^{-i / 2} h\left(m\left(B\left(C, 2^{(-i+5) / 2}\right)\right)\right) \leq K q^{3 / 4}
$$

So, by change of index,

$$
\sum_{i=1}^{q-5} 2^{-i / 2} h\left(m\left(B\left(C, 2^{-i / 2}\right)\right)\right) \leq K q^{3 / 4} .
$$

If $\lambda\left(C \triangle C^{\prime}\right) \leq 4\left(2^{-q}\right)$, then $\phi\left(C^{\prime}\right)=C$. So $\phi\left(B\left(C, 2\left(2^{-q / 2}\right)\right)\right) \subset B\left(C, 2\left(2^{-q / 2}\right)\right)$ and

$$
h\left(m\left(B\left(C, 2\left(2^{-q / 2}\right)\right)\right)\right) \leq h\left(m_{q}\left(B\left(C, 2\left(2^{-q / 2}\right)\right)\right)\right) .
$$

Since $2^{-q / 2} h\left(m_{q}\left(B\left(C, 2\left(2^{-q / 2}\right)\right)\right)\right) \leq K q^{3 / 4}$ from (18), we get

$$
\sum_{i=1}^{q-2} 2^{-i / 2} h\left(m\left(B\left(C, 2^{-i / 2}\right)\right)\right) \leq K q^{3 / 4} .
$$

For $C$ in $T$, the ball $B\left(C, 2^{-(q-2) / 2}\right)$ (considered as a ball in $T$ ) is reduced to $C$. We have proved that the quantity (13) (where $l$ is the smallest integer $\geq(q-2) / 2$ ) 
is $\leq K q^{-3 / 4}$. Application of Lemma 3 , where $X_{1}, \ldots, X_{n}$ are fixed such that the conditions of Lemma 1 occur give, for some universal constants $K, \beta$,

$$
\begin{gathered}
Q\left(\operatorname{Sup}_{C, C^{\prime} \in T}\left|\frac{1}{n} \sum_{i \leq n} \varepsilon_{i}(\omega) 1_{X_{i}}(C)-\sum_{i \leq n} \varepsilon_{i}(\omega) 1_{X_{i}}\left(C^{\prime}\right)\right| \geq K n^{-1 / 2}(\log n)^{3 / 4}\right) \\
\leq 2 \exp \left(-\beta(\log n)^{3 / 2}\right) .
\end{gathered}
$$

We can surely arrange that $T$ contains the empty lower set, so we get

$$
\begin{gathered}
Q\left(\operatorname{Sup}_{C \in T}\left|\frac{1}{n} \sum_{i \leq n} \varepsilon_{i}(\omega) 1_{X_{i}}(C)\right| \geq K n^{-1 / 2}(\log n)^{3 / 4}\right) \\
\leq 2 \exp \left(-\beta(\log n)^{3 / 2}\right) .
\end{gathered}
$$

Combining this with Lemma 2 and Lemma 1 yields Theorem B.

3. Construction of the majorizing measure. The measure $m_{q}$ will be constructed inductively. The computations require great care, but the idea is very simple. A given function $u$ in $\mathcal{F}_{q+1}$ is made from two simpler pieces $u_{1}$ and $u_{2}$ in $\mathcal{F}_{q}$, properly scaled and glued together at the point $\left(\frac{1}{2}, u\left(\frac{1}{2}\right)\right)$. Given $u_{1}$ and $u_{2}$ in $\mathcal{F}_{q},(q \geq 1)$, and $x$ in $[0,1]$, we define $u=f\left(u_{1}, u_{2}, x\right)$ in $\mathcal{F}_{q+1}$ in the following way. For $0 \leq t \leq 1 / 2$, we set

$$
u(t)=1-(1-x)\left(1-u_{1}(2 t)\right)=x+(1-x) u_{1}(2 t) .
$$

For $1 / 2 \leq t \leq 1$, we set

$$
u(t)=x u_{2}(2 t-1) .
$$

In particular, $u\left(\frac{1}{2}\right)=x$. The basic idea is that the elements of $\mathcal{F}_{q}$ that are really important are those for which $\left|u\left(\frac{1}{2}\right)-\frac{1}{2}\right|$ is of order $1 / \sqrt{q}$. So, when $F_{q}$ is provided with $m_{q}$, the law of $u \rightarrow u\left(\frac{1}{2}\right)$ should be basically concentrated on the interval $1 / 2-1 / \sqrt{q}, 1 / 2+1 / \sqrt{q}$. A simple way to achieve this is by requiring this law to be the probability $\nu_{q}$ on $[0,1]$, of density $\gamma_{q} /(1+|x-1 / 2| \sqrt{q})^{3}$, where

$$
\gamma_{q}^{-1}=\int_{0}^{1}(1+|x-1 / 2| \sqrt{q})^{-3} d x=\frac{1}{\sqrt{q}}\left(1-\frac{1}{(1+\sqrt{q} / 2)^{2}}\right) .
$$

The exact value of $\gamma_{q}$ is irrelevant; we will only use that $\gamma_{q}$ is of order $\sqrt{q}$.

For $q=1, u$ in $\mathcal{F}_{q}$ is determined by $u\left(\frac{1}{2}\right)$, so $m_{1}$ is already determined. Assuming now that $m_{q}$ has been constructed, we consider the product measure $\delta_{q}=m_{q} \otimes$ $m_{q} \otimes \nu_{q+1}$ on $\mathcal{F}_{q} \otimes \mathcal{F}_{q} \otimes[0,1]$, and we take for $m_{q+1}$ the image measure of $\delta_{q}$ by the map $\left(u_{1}, u_{2}, x\right) \rightarrow f\left(u_{1}, u_{2}, x\right)$. In other words, if $G$ is a Borel subset of $\mathcal{F}_{q+1}$, and if

$$
H=\left\{\left(u_{1}, u_{2}, x\right) ; f\left(u_{1}, u_{2}, x\right) \in G\right\}
$$

we set

$$
m_{q+1}(G)=\iiint_{H} d m_{q}\left(u_{1}\right) d m_{q}\left(u_{2}\right) d \nu_{q+1}(x)
$$

The complicated nature of $m_{q}$ makes the numbers $m_{q}\left(B_{i, q}(u, 1)\right)$ hard to evaluate, so a better approach is to try to prove (17) by induction over $q$. In order to be 
able to carry on the induction, we will unfortunately be forced to consider a more complicated induction hypothesis. This hypothesis (equation (31)) will involve a sequence of parameters $\left(\theta_{k}\right)$ and related quantities $a_{k, i}$. To prove (17), we will need only to know that the induction hypothesis holds when $\theta_{k}=1$ for all $k$. The proof contains lots of elementary, but lengthy and uninspiring estimates. To get the overall idea, one should first read the proof of the crucial estimate (29) and the ensuing comment. This will provide motivation for the computational Lemmas 6 and 7 , and for the choice of the induction hypothesis.

Throughout the proof, we use the following functions $\alpha, \beta$ on $[0,1]$. We define

$$
\begin{array}{ll}
\alpha(x)=5 / 6 & \text { for } 0 \leq x \leq 1 / 4, \\
\alpha(x)=(3-2 x) / 4(1-x) & \text { for } 1 / 4 \leq x \leq 3 / 4, \\
\alpha(x)=3 / 2 & \text { for } x \geq 3 / 4 .
\end{array}
$$

So $5 / 6 \leq \alpha(x) \leq 3 / 2$. We set $\beta(x)=\alpha(1-x)$, so $\beta(x)=(2 x+1) / 4 x$ for $1 / 4 \leq$ $x \leq 3 / 4$. We note that $(1-x) \alpha(x)+x \beta(x) \leq 1$ for each $x$.

LEMMA 6. Let $q \geq 1,0<b \leq 1, a \geq 0,2 a \leq b$. Let $u_{1}, u_{2}, v_{1}, v_{2}$ in $\mathcal{F}_{q}$. Let $i \leq q$. Assume

$$
\begin{gathered}
v_{1} \in B_{i, q}\left(u_{1}, \alpha(x)(b-2 a)\right), \\
v_{2} \in B_{i, q}\left(u_{2}, \beta(x)(b-2 a)\right)
\end{gathered}
$$

and $|x-y| \leq 2^{-i} a$. Let $u=f\left(u_{1}, u_{2}, x\right), v=f\left(v_{1}, v_{2}, y\right)$.

Then $v \in B_{i+1, q+1}(u, b)$.

ProOF. This is indeed a simple algebraic computation. From (23), (24) and the definition of $B_{i, q}$, we get

$$
\sum_{d \in A_{i}}\left|v_{1}(d)-u_{1}(d)\right| \leq \alpha(x)(b-2 a)
$$

and

$$
\sum_{d \in A_{i}}\left|v_{2}(d)-u_{2}(d)\right| \leq \beta(x)(b-2 a) .
$$

We have to evaluate $\sum_{d \in A_{i+1}}|u(d)-v(d)|$. We break the sum in two parts. For $d$ in $A_{i+1}, d<1 / 2$, we have $2 d \in A_{i}$. We note that

$$
\begin{aligned}
|u(d)-v(d)| & =\left|x+(1-x) u_{1}(2 d)-y-(1-y) v_{1}(2 d)\right| \\
& \leq(1-x)\left|u_{1}(2 d)-v_{1}(2 d)\right|+\left|(x-y)\left(1-v_{1}(2 d)\right)\right| \\
& \leq(1-x)\left|u_{1}(2 d)-v_{1}(2 d)\right|+|x-y| \\
& \leq(1-x)\left|u_{1}(2 d)-v_{1}(2 d)\right|+2^{-i} a .
\end{aligned}
$$

So we get

$$
\sum_{\substack{d<1 / 2 \\ d \in A_{i+1}}}|v(d)-u(d)| \leq(1-x) \alpha(x)(b-2 a)+\left(2^{i}-1\right) 2^{-i} a .
$$

For $d$ in $A_{i+1}, d>1 / 2$, we have $2 d-1 \in A_{i}$. We note that

$$
\begin{aligned}
|u(d)-v(d)| & =\left|x u_{2}(2 d-1)-y v_{2}(2 d-1)\right| \\
& \leq x\left|u_{2}(2 d-1)-v_{2}(2 d-1)\right|+\left|(x-y) v_{2}(2 d-1)\right| \\
& \leq x\left|u_{2}(2 d-1)-v_{2}(2 d-1)\right|+2^{-i} a .
\end{aligned}
$$


So we get

$$
\sum_{\substack{d>1 / 2 \\ d \in A_{i+1}}}|v(d)-u(d)| \leq x \beta(x)(b-2 a)+\left(2^{i}-1\right) 2^{-i} a .
$$

So we get, since $\left|v\left(\frac{1}{2}\right)-u\left(\frac{1}{2}\right)\right|=|x-y| \leq 2^{-i} a$,

$$
\sum_{d \in A_{i+1}}|v(d)-u(d)| \leq(b-2 a)(x \beta(x)+(1-x) \alpha(x))+2 a
$$

and the result follows from $x \beta(x)+(1-x) \alpha(x) \leq 1$.

We fix now a number $\tau>1$. (The precise value that this parameter has to take will be determined in Lemma 9.) We set $g(t)=h^{2}(t)=\log (1 / t)$ for $0<t \leq 1$. Fix a number $\theta>0$, and let $i_{0}$ be the largest integer such that $2^{-i_{0}} \theta \geq q^{-1 / 2}$, so $2^{-i_{0}} \theta \leq 2 q^{-1 / 2}$. We set

$$
a_{i}=2^{-\left|i-i_{0}\right| / 4-\tau} .
$$

The fact that $a_{i} \geq 2^{-|i-j| / 4} a_{j}$ for all $i, j$ will be used constantly.

LEMMA 7. For some universal constant $K$, we have

$$
\sum_{i=1}^{q} 2^{-i} g\left(\nu_{q}\left(\left[x-\theta 2^{-i} a_{i}, x+\theta 2^{-i} a_{i}\right]\right)\right) \leq(K / \theta)\left[q^{-1 / 2}+q^{1 / 2}(x-1 / 2)^{2}\right] .
$$

ProOF. Case 1. $|x-1 / 2| \leq q^{-1 / 2}$. Let $i_{1}=i_{0}-\left(\frac{4}{3}\right)(\tau+1)$. For $i \leq i_{1}$, we have

$$
\begin{aligned}
\theta 2^{-i} a_{i} & =2^{i_{0}-i} a_{i}\left(\theta 2^{-i_{0}}\right) \geq q^{-1 / 2} 2^{i_{0}-i} a_{i} \geq q^{-1 / 2} 2^{i_{0}-i} 2^{-\left|i_{0}-i\right| / 4} a_{i_{0}} \\
& \geq q^{-1 / 2} 2^{3 / 4\left(i_{0}-i\right)} 2^{-\tau} \geq 2 q^{-1 / 2} .
\end{aligned}
$$

Let $u_{i}=2^{3 / 4\left(i_{0}-i\right)-\tau-1}$ (so $u_{i} \geq 1$ ), and let

$$
\begin{aligned}
J_{i} & =\left[1 / 2-q^{-1 / 2} u_{i}, 1 / 2+q^{-1 / 2} u_{i}\right], \\
I_{i} & =\left[x-\theta 2^{-i} a_{i}, x+\theta 2^{-i} a_{i}\right] .
\end{aligned}
$$

Since $\mid x-1 / 2] \leq q^{-1 / 2}$, we have $J_{i} \subset I_{i}$. We have

$$
1-\nu_{q}\left(J_{i}\right) \leq K \int_{u_{i}}^{\infty} \frac{q^{1 / 2}}{\left(1+t q^{1 / 2}\right)^{3}} d t \leq K u_{i}^{-2} .
$$

Also, for some universal constant $\alpha, \nu_{q}\left(J_{i}\right) \geq \alpha$. Since $g(t) \leq K(1-t)$ for $t \geq \alpha$, we get $g\left(\nu_{q}\left(J_{i}\right)\right) \leq K u_{i}^{-2}$. It follows that

$$
\begin{aligned}
\sum_{i=1}^{i_{1}} 2^{-i} g\left(\nu_{q}\left(I_{i}\right)\right) & \leq K \sum_{i=1}^{i_{1}} 2^{-i} 2^{-3\left(i_{0}-i\right) / 2} \\
& \leq K 2^{-i_{0}} \sum_{i=1}^{i_{1}} 2^{-\left(i_{0}-i\right) / 2} \leq K^{\prime} 2^{-i_{0}} \leq \frac{2 K^{\prime} q^{-1 / 2}}{\theta}
\end{aligned}
$$

For $i \geq i_{1}$, we have $\theta 2^{-i} a_{i} \leq K q^{-1 / 2}$, so since $|x-1 / 2| \leq q^{-1 / 2}$, the density of $\nu_{q}$ on the interval $I_{i}$ is greater than $q^{1 / 2} / K$, so

$$
\begin{aligned}
\nu_{q}\left(I_{i}\right) & \geq q^{1 / 2} \theta 2^{-i} a_{i} / K \geq 2^{i_{0}-i} a_{i} / K \geq 2^{i_{0}-i} 2^{-\left|i_{0}-i\right| / 4} 2^{-\tau} / K \\
& \geq 2^{-5\left|i-i_{0}\right| / 4} / K .
\end{aligned}
$$


It follows that

$$
\begin{aligned}
\sum_{i \geq i_{1}} 2^{-i} g\left(\nu_{q}\left(I_{i}\right)\right) & \leq K \sum_{i \geq i_{1}}\left(2^{-i}\left(\left|i-i_{0}\right|+1\right)\right) \\
& \leq K 2^{-i_{1}} \leq K^{\prime} 2^{-i_{0}} \leq 2 K^{\prime} q^{-1 / 2} / \theta
\end{aligned}
$$

This finishes the proof in that case.

Case 2. $|x-1 / 2| \geq q^{-1 / 2}$.

Let $i_{1}$ be the largest integer such that $\theta 2^{-i_{1}} a_{i_{1}} \geq|x-1 / 2|$. We have

$$
\theta 2^{-i_{0}} a_{i_{0}} \leq 2 q^{-1 / 2} 2^{-\tau} \leq 2^{1-\tau}|x-1 / 2| .
$$

Since we assume $\tau>1$, it follows that $i_{1} \leq i_{0}$. For $i \leq i_{1}$, we have

$$
\begin{aligned}
\theta 2^{-i} a_{i} & \geq \theta 2^{-i} 2^{-\left|i-i_{1}\right| / 4} a_{i_{1}} \geq \theta 2^{-i_{1}} a_{i_{1}} 2^{3\left(i_{1}-i\right) / 4} \\
& \geq 2^{3\left(i_{1}-i\right) / 4}|x-1 / 2| .
\end{aligned}
$$

Let $u_{i}=2^{3\left(i_{1}-i\right) / 4-1}$, so $u_{i} \geq 1$ for $i \leq i_{1}-2$. So, for $i \leq i_{1}-2$,

$$
I_{i}=\left[x-\theta 2^{-i} a_{i}, x+\theta 2^{-i} a_{i}\right] \supset\left[1 / 2-u_{i} q^{-1 / 2}, 1 / 2+u_{i} q^{-1 / 2}\right] .
$$

The same computation as in Case 1 shows that

$$
\sum_{i \leq i_{1}-2} 2^{-i} g\left(\nu_{q}\left(I_{i}\right)\right) \leq K 2^{-i_{1}}
$$

For $i \geq i_{1}-1$, we have

$$
\begin{aligned}
\theta 2^{-i} a_{i} & \leq \theta 2^{-i_{1}-1} a_{i_{1}+1}\left(2^{-i+i_{1}+1} 2^{\left|i-i_{1}-1\right| / 4}\right) \\
& \leq|x-1 / 2| 2^{5 / 2} \leq 2^{3}|x-1 / 2|
\end{aligned}
$$

It follows that the interval $I_{i}$ contains an interval of length $\geq \theta 2^{-i-3} a_{i}$ on which the density of $\nu_{q}$ is at least the density of $\nu_{q}$ at $x$. So we have

$$
\nu_{q}\left(I_{i}\right) \geq \theta 2^{-i} a_{i} q^{1 / 2}\left(1+q^{1 / 2}|x-1 / 2|\right)^{-3} / K \text {. }
$$

Now

$$
\theta 2^{-i} a_{i} \geq \theta 2^{-i_{1}} a_{i_{1}} 2^{-i+i_{1}} 2^{-\left|i-i_{1}\right| / 4} \geq|x-1 / 2| 2^{-5\left|i-i_{1}\right| / 4} .
$$

So, we find

$$
\nu_{q}\left(I_{i}\right) \geq 2^{-5\left|i-i_{1}\right| / 4}\left(q^{1 / 2}|x-1 / 2|\right)^{-2} / K
$$

It follows that

$$
\sum_{i \geq i_{1}-1} 2^{-i} g\left(\nu_{q}\left(I_{i}\right)\right) \leq K 2^{-i_{1}}\left(1+\log \left(q^{1 / 2}|x-1 / 2|\right)\right)
$$

and also

$$
\sum_{i \leq q} 2^{-q} g\left(\nu_{q}\left(I_{i}\right)\right) \leq K 2^{-i_{1}}\left(1+\log \left(q^{1 / 2}|x-1 / 2|\right)\right)
$$

We note that

$$
\begin{aligned}
|x-1 / 2| & \geq \theta 2^{-i_{1}-1} a_{i_{1}+1} \geq \theta 2^{-i_{0}} 2^{i_{0}-i_{1}-1} 2^{-\left|i_{0}-i_{1}-1\right| / 4} a_{i_{0}} \\
& \geq q^{-1 / 2} 2^{3\left(i_{0}-i_{1}\right) / 4-2-\tau} .
\end{aligned}
$$

So,

$$
2^{i_{0}-i_{1}} \leq K\left(q^{1 / 2}|x-1 / 2|\right)^{4 / 3}
$$


Using the inequality $\log t \leq K t^{2 / 3}$, for $t \geq 1$, we see that

$$
\begin{aligned}
2^{-i_{1}} & \left(1+\log \left(q^{1 / 2}|x-1 / 2|\right)\right) \\
& \leq K 2^{-i_{0}}\left(q^{1 / 2}|x-1 / 2|\right)^{4 / 3}\left(1+\log \left(q^{1 / 2}|x-1 / 2|\right)\right) \\
& \leq\left(K q^{-1 / 2} / \theta\right)\left(q^{1 / 2}|x-1 / 2|\right)^{2} .
\end{aligned}
$$

This completes the proof.

PROPOSITION 8. Let $q \geq 1$. Let $\theta>0$, let $i_{0}$ be the largest integer with $2^{-i_{0}} \theta \geq(q+1)^{-1 / 2}$. Set $a_{i}=2^{-\left|i-i_{0}\right| / 4-\tau}$. For $i \leq q+1$, let $\xi_{i}>0$ with $\xi_{i}>a_{i}$. Let $u$ in $\mathcal{F}_{q+1}$. Let $x=u\left(\frac{1}{2}\right)$, and let $u_{1}, u_{2}$ in $\mathcal{F}_{q}$ with $u=f\left(u_{1}, u_{2}, x\right)$. Then the following holds:

$$
\begin{array}{rl}
\sum_{i=1}^{q+1} 2^{-i} & g\left(m_{q+1}\left(B_{i, q+1}\left(u, \theta \xi_{i}\right)\right)\right) \\
\leq & \frac{1}{2} \sum_{i=1}^{q} 2^{-i} g\left(m_{q}\left(B_{i, q}\left(u_{1}, \alpha(x) \theta\left(\xi_{i+1}-a_{i+1}\right)\right)\right)\right) \\
& +\frac{1}{2} \sum_{i=1}^{q} 2^{-i} g\left(m_{q}\left(B_{i, q}\left(u_{2}, \beta(x) \theta\left(\xi_{i+1}-a_{i+1}\right)\right)\right)\right) \\
& +(K / \theta)\left(q^{-1 / 2}+q^{1 / 2}|x-1 / 2|^{2}\right) .
\end{array}
$$

PROOF. It follows from Lemma $6{ }^{*}$, with $b=\theta \xi_{i+1}, a=\theta a_{i+1} / 2$ and from (22) that for $1 \leq i \leq q$, we have

$$
\begin{gathered}
m_{q+1}\left(B_{i+1, q+1}\left(u, \theta \xi_{i+1}\right)\right) \geq m_{q}\left(B_{i, q}\left(u_{1}, \alpha(x) \theta\left(\xi_{i+1}-a_{i+1}\right)\right)\right), \\
m_{q}\left(B_{i, q}\left(u_{2}, \beta(x) \theta\left(\xi_{i+1}-a_{i+1}\right)\right)\right) \nu_{q+1}\left(\left[x-2^{-i-1} \theta a_{i+1}, x+2^{-i-1} \theta a_{i+1}\right]\right) .
\end{gathered}
$$

We also have

$$
m_{q+1}\left(B_{1, q+1}\left(u, \theta \xi_{1}\right)\right) \geq \nu_{q+1}\left(\left[x-2^{-1} \theta a_{1}, x+2^{-1} \theta a_{1}\right]\right) .
$$

Now $g$ is decreasing and $g(u v w)=g(u)+g(v)+g(w)$, so we get the result from Lemma 7 , by noting that $(q+1)^{1 / 2} \leq 2 q^{1 / 2}$.

Comment. The idea is now to apply (29) again to each of the sums in the right side of (29). If we could prove that (29) holds for $\xi_{i}=1$ without the term $-a_{i+1}$ in the radius of the balls on the right side, together with Lemma 10 we could prove by induction over $q$ that

$$
\sum_{i=1}^{q} 2^{-i} g\left(m_{q}\left(B_{i, q}(u, \theta)\right)\right) \leq \frac{K q^{1 / 2}}{\theta} .
$$

But we must take care that the perturbations that occur at each step can be controlled. This is the object of Lemma 9. We also have to use as induction hypothesis a statement in which the perturbations already occur, so that application of (29) does not change its form, and this is what motivates (31).

LEMMA 9. There exists $\tau>0$ with the following property. Consider a sequence $\left(\theta_{k}\right)_{k \geq q+1}$ such that for each $k \geq q+1,0<\frac{2}{3} \theta_{k} \leq \theta_{k+1} \leq \frac{6}{5} \theta_{k}$. Denote by $i_{k}$ the 
largest integer with $\theta_{k} 2^{-i_{k}} \geq k^{-1 / 2}$. Set $a_{k, i}=2^{-\left|i-i_{k}\right| / 4-\tau}$. Then for $j \geq 1$, we have

$$
\sum_{l \geq 0} a_{q+l, j+l} \leq \frac{1}{2}
$$

PROOF. We have $2^{i_{k}} k^{-1 / 2} \leq \theta_{k} \leq 2^{i_{k}+1} k^{-1 / 2}$. For $n \geq k \geq q+1$, we have

$$
2^{i_{n}-i_{k}-1}(k / n)^{1 / 2} \leq \theta_{n} / \theta_{k} \leq 2^{i_{n}-i_{k}+1}(k / n)^{1 / 2} \text {. }
$$

So we have $\frac{1}{2}(2 / 3)^{n-k} \leq 2^{i_{n}-i_{k}}$ and

$$
2^{i_{n}-i_{k}} \leq 2(n / k)^{1 / 2}(6 / 5)^{n-k} \leq 2(1+n-k)^{1 / 2}(6 / 5)^{n-k} .
$$

Since $2 / 3>1 / 2$ and $6 / 5<2$, it follows easily that for some $\delta<1$, we have $\left|i_{n}-i_{k}\right| \leq K+\delta|n-k|$. It follows that for any $j$, there are at most $1+K /(1-\delta)$ values of $l$ for which $j+l-i_{q+l}$ is a given integer. Since the series $\sum_{i \in \mathbf{Z}} 2^{-|i| / 4}$ converges, it is enough to take $\tau$ large enough that

$$
2^{-\tau}\left(1+\frac{K}{1-\delta}\right) \sum_{i \in \mathbf{Z}} 2^{-|i| / 4} \leq \frac{1}{2}
$$

LEMMA 10. For $x$ in $[0,1]$, we have

$$
\frac{1}{2}(1 / \alpha(x)+1 / \beta(x)) \leq 1-\left(x-\frac{1}{2}\right)^{2} / 4 .
$$

PROOF. Letting $x=1 / 2+t$, for $-1 / 4 \leq t \leq 1 / 4$, we have

$$
\frac{1}{2}(1 / \alpha(x)+1 / \beta(x))=\left(1-2 t^{2}\right) /\left(1-t^{2}\right) \leq 1-t^{2} .
$$

For $|t| \geq 1 / 4$, we have, since $|t| \leq 1 / 2$,

$$
\frac{1}{2}(1 / \alpha(x)+1 / \beta(x))=\frac{1}{2}(2 / 3+6 / 5)=14 / 15 \leq 1-1 / 16 \leq 1-t^{2} / 4 .
$$

This completes the proof.

We denote now by $K_{0}$ the constant of Proposition 8 . We note the following:

$$
\text { For } q \geq 1, \quad q^{1 / 2}+\frac{1}{3} q^{-1 / 2} \leq(q+1)^{1 / 2} .
$$

We now prove the main result.

PROPOSITION 11. Consider a sequence $\left(\theta_{k}\right)_{k \geq q}$ such that for each $k$,

$$
\frac{2}{3} \theta_{k} \leq \theta_{k+1} \leq \frac{6}{5} \theta_{k} \text {. }
$$

Define $a_{k, i}$ as in Lemma 9. Then we have for each $u$ in $\mathcal{F}_{q}$

$$
\sum_{i=1}^{q} 2^{-i} g\left(m_{q}\left(B_{i, q}\left(u, \theta_{q}\left(1-\sum_{l \geq 1} a_{q+l, i+l}\right)\right)\right) \leq \frac{K_{1} q^{1 / 2}}{\theta_{q}}\right.
$$

ProOF. We note that the numbers $a_{q+l, i+l}, l \geq 1$, depend only on $\theta_{q+1}$, $\theta_{q+2}, \ldots$, but not on $\theta_{q}$. The proof goes by induction over $q$. For $q=1$, we note that from Lemma 9 , we have $\sum_{l \geq 1} a_{1+l, 1+l} \leq 1 / 2$. Since $\nu_{1}$ has a density bounded below on $[0,1]$, we have

$$
m_{1}\left(B_{1,1}\left(u, \theta_{1}\left(1-\sum_{l \geq 1} a_{1+l, 1+l}\right)\right)\right) \geq \frac{\theta_{1}}{K}
$$


if $\theta_{1} \leq 2$, and this measure is 1 for $\theta_{1} \geq 2$. So (31) holds for $q=1$ if $K_{1}$ is large enough that $\theta_{1} g\left(K / \theta_{1}\right) \leq K_{1}$ for $0<\theta_{1} \leq 2$. We now fix $K_{1} \geq 4 K_{0}$ with that property, so (31) holds for $q=1$. We now prove (31) by induction over $q$. Assuming that it holds for $q$, we prove it for $q+1$. We note that

$$
\sum_{l \geq 1} a_{q+1+l, i+1+l}+a_{q+1, i+1}=\sum_{l \geq 1} a_{q+l, i+l} .
$$

We use Proposition 8 with $\theta=\theta_{q+1}, \xi_{i}=1-\sum_{l \geq 1} a_{q+1+l, i+l}, a_{i}=a_{q+1, i}$ (so $a_{i}<\xi_{i}$ by Lemma 9) and we get (with $x=u\left(\frac{1}{2}\right), u=f\left(u_{1}, u_{2}, x\right), u_{1}$ and $u_{2}$ in $\left.\mathcal{F}_{q}\right)$

$$
\begin{aligned}
& \sum_{i=1}^{q+1} 2^{-i} g\left(m_{q+1}\left(B_{i, q+1}\left(u, \theta_{q+1}\left(1-\sum_{l \geq 1} a_{q+1+l, i+l}\right)\right)\right)\right) \\
& \leq \frac{1}{2} \sum_{i=1}^{q} 2^{-i} g\left(m_{q}\left(B_{i, q}\left(u_{1}, \alpha(x) \theta_{q+1}\left(1-\sum_{l \geq 1} a_{q+l, i+l}\right)\right)\right)\right) \\
&+\frac{1}{2} \sum_{i=1}^{q} 2^{-i} g\left(m_{q}\left(B_{i, q}\left(u_{2}, \beta(x) \theta_{q+1}\left(1-\sum_{l \geq 1} a_{q+l, i+l}\right)\right)\right)\right) \\
&+\left(K_{0} / \theta_{q+1}\right)\left(q^{-1 / 2}+q^{1 / 2}|x-1 / 2|^{2} .\right.
\end{aligned}
$$

Since $5 / 6 \leq \alpha(x), \beta(x) \leq 3 / 2$, we can use the induction hypothesis with $\theta_{q}=$ $\alpha(x) \theta_{q+1}$ (resp. $\theta_{q}=\beta(x) \theta_{q+1}$ ) and we find that this quantity is less than

$$
\begin{aligned}
\left(K_{1} q^{1 / 2} / 2 \theta_{q+1}\right)(1 / \alpha(x)+1 / \beta(x))+\left(K_{1} / 4 \theta_{q+1}\right)\left(q^{-1 / 2}+q^{1 / 2}|x-1 / 2|^{2}\right) \\
\quad \leq\left(K_{1} / \theta_{q+1}\right)\left(q^{1 / 2}-q^{1 / 2}|x-1 / 2|^{2} / 4+q^{-1 / 2} / 4+q^{1 / 2}|x-1 / 2|^{2} / 4\right) \\
\quad \leq\left(K_{1} / \theta_{q+1}\right)\left(q^{1 / 2}+q^{-1 / 2} / 4\right) \leq K_{1}(q+1)^{1 / 2} / \theta_{q+1}
\end{aligned}
$$

using Lemma 10 and (30). The proof is complete.

We now prove Proposition 4 . Taking $\theta_{k}=1$ for each $k$, we get

$$
\sum_{i=1}^{q} 2^{-i} g\left(m_{q}\left(B_{i, q}\left(u,\left(1-\sum_{l \geq 1} a_{q+l, i+l}\right)\right)\right)\right) \leq K_{1} q^{1 / 2} .
$$

So, since $g$ is decreasing

$$
\sum_{i=1}^{q} 2^{-i} g\left(m_{q}\left(B_{i, q}(u, 1)\right)\right) \leq K_{1} q^{1 / 2} .
$$

Now we have by Cauchy-Schwarz,

$$
\begin{gathered}
\sum_{i=1}^{q} 2^{-i / 2} h\left(m_{q}\left(B_{i, q}(u, 1)\right)\right) \leq q^{1 / 2}\left(\sum_{i=1}^{q} 2^{-i} g\left(m_{q}\left(B_{i, q}(u, 1)\right)\right)\right)^{1 / 2} \\
\leq q^{1 / 2}\left(K_{1} q^{1 / 2}\right)^{1 / 2} \leq K q^{3 / 4}
\end{gathered}
$$

This finishes the proof. 


\section{REFERENCES}

1. R. M. Dudley, The size of compact subsets of Hilbert space and continuity of Gaussian processes, J. Funct. Anal. 1 (1967), 290-330.

2. _ A course on empirical processes, Lecture Notes in Math., vol. 1097, Springer-Verlag, 1982.

3. Empirical and Poisson processes on classes of sets too large for the central limit theorem, Z. Wahrsch. Verw. Gebiete 61 (1983), 355-368.

4. X. Fernique, Régularité des trajectoires des fonctions aléatoires Gaussiennes, Lecture Notes in Math., vol. 480, Springer-Verlag, 1974, pp. 1-96.

5. E. Giné and J. Zinn, Some limit theorems for empirical processes, Ann. Probab. 12 (1984), 929-989.

6. R. M. Karp, M. Luby, and A. Marchetti-Spaccamela, Probabilistic analysis of multidimensional bin packing problems, Proc. Sixteenth Annual ACM Sympos. on Theory of Computing, Washington, D. C., 1984, pp. 289-298.

7. F. T. Leighton and P. Shor, Tight bounds for minimax grid matching with applications to the average case analysis of algorithms, Proc. Eighteenth Annual ACM Sympos. on Theory of Computing, May, 1986, pp. 91-103.

8. P. W. Shor, The average-case analysis of some on-line algorithms for bin packing, Proc. Twentyfifth Annual Sympos. on Foundations of Computer Science, Singer Island, Florida, 1984, pp. 193-200.

9. M. Talagrand, Regularity of Gaussian processes, Acta Math. (to appear).

Faculty of Management Sciences, The Ohio State University, 1775 College RoAD, Columbus, OHIO 43210

Equipe D'ANalyse-Tour 46, Université Paris VI, 4 Place Jussieu, 75230 Paris CEDEX 05, FRANCE

Department of Mathematics, The Ohio State University, 231 W. 18th St., COlumbus, OHIO 43210 